\title{
Four decades of studies on population aging in China
}

\author{
Jiehua Lu ${ }^{1} \cdot$ Qin Liu ${ }^{1}$ \\ Received: 20 March 2019 / Accepted: 9 April 2019 / Published online: 16 April 2019 \\ (C) China Population and Development Research 2019
}

\begin{abstract}
The last 4 decades of reform and opening up have not only witnessed the rapid development of China's social economy, but have also marked the crucial transition of China's demographic structure from one that is mature to one that is old. As China's population has aged rapidly in recent years, studies of aging have made worthwhile achievements documenting the process. This article mainly summarizes the development of studies on aging in China during the past 40 years and finds that research into the topic of aging during these years can be divided into three phases. The phases are characterized by particular kinds of research. Phase one, the study of aging trends and causes; phase two, the study of aging characteristics and policy responses; and phase three, the study of top-level design of aging policies and programs, and the development of integrated interdisciplinary research efforts. The five main characteristics of China's studies on aging during the past 4 decades and five future prospects for studies on aging are discussed at the end of this paper.
\end{abstract}

Keywords Reform and opening up - Studies on population aging · Topic phases · Future prospects

\section{Introduction}

The last 4 decades of reform and opening up were not only a time of rapid development of China's social economy, but also witnessed the crucial transition of China's demographic structure from one that is mature to one that is old. As China's population has aged during the past 4 decades, studies of the aging process have produced worthwhile results. Research on population aging is important because it can help us to understand correctly the main characteristics and the typical development of the aging population. Additionally, research on population aging can scientifically predict the population structure and aging trends, and can accurately assess the multiple influences of aging on various aspects of social, economic, cultural, technological

Qin Liu

liuqin1991@pku.edu.cn

1 Department of Sociology, Peking University, Beijing, China 
and political development. Given the wide range of issues they consider, studies on population aging are valuable references for decision-making and provide technical support to aid in the formulation of scientific aging policies and in coping with problems that arise from population aging.

In the context of the background of China's population aging, this article summarizes the results of China's studies on population aging during the last $4 \mathrm{dec}-$ ades, illuminating the development process comprehensively and dynamically. In conclusion, the article looks ahead by highlighting the creative characteristics of the research on aging we can expect to see in the near future.

\section{The background of population aging in China during the 4 decades of reform and opening up}

The combination of declining birth rates and increasing average life expectancy led to an accelerated aging process in China. After 40 years of reform and opening up, China completed a new phase of demographic transformation that changed the demographic structure of the country from the mature to the old.

Data from the National Bureau of Statistics shows that the number and proportion of the elderly population aged 65 and above in China has increased continuously since 1978, with the growth rate showing a trend of first fast, then slow and then gradually accelerated during the 4 decades. Changes in the number of elderly people aged 65 and above during the 40 years of reform and opening suggest that the evolution of China's aging population can be roughly divided into three stages: the accumulative stage, the initial stage, and the accelerating stage. During the accumulative stage (1978-1995), the elderly population aged 65 and above showed a cumulative growth trend, with an average annual growth rate of 3.19\%. During the initial stage (1996-2000), the aging population kept growing, with an average annual growth rate of $2.97 \%$. By 2000 , the total number of elderly people aged 65 and above had reached 88.21 million, accounting for $7 \%$ of China's total population. At this point, China began to become an aging society. During the acceleration stage (2001-2018), the first baby boom population born soon after the founding of the People's Republic of China in 1949 became the elderly. In this stage, the annual growth rate of the elderly population aged 65 and above reached $3.28 \%$, significantly exceeding the annual growth rate of $0.66 \%$ for the total population.

In the years to come, China's population of the elderly is expected to continue developing rapidly and the aging process to continue speeding up. According to the projection on the trend of the China's population aging in the twenty-first century, in the first half of the century, the size of China's elderly population will keep rising at a relatively fast rate, and then in the second half of the century begin to decline at a slow rate. The size of the elderly population aged 60 and above is expected to peak in the twenty-first century at about 482 million, probably in the year 2053 (Zhai et al. 2017).

China's elderly population accounts for one fifth of the world's total elderly population, so the aging of China's population is of great importance to the aging process of the global population. The aging of China's population is having a profound 
impact on China's economic, social, political, cultural, scientific and technological development, and is putting great pressure on systems that deliver old-age care, medical care, and social services. Population aging will be a basic national condition of China throughout the twenty-first century. Developing an active response to population aging is a long-term strategic task for the country.

Since 1996, after China's "Law on the Protection of the Rights and Interests of the Elderly" came into effect, a succession of aging policies have been put forward. Initially, the government introduced a number of policies, including ones to promote elderly welfare, to provide support services for elderly people, and to provide preferential treatment for the elderly. Later policies concerning the rural five guarantees, pension services and pension institutions were implemented. Recently, the focus of policies has been on urban and rural pension support, elderly housing, the development of appropriate living environments for the elderly and programs that combine medical treatment and pension support. Clearly, national policies play an indispensable role in the process of aging development. The reform and development of programs for China's elderly and old-age care service system were important parts of the 11th Five-Year Plan (2016-2010) and 12th Five-Year Plan (2011-2015) in China, and continue to be important in the 13th Five-Year Plan (2016-2020). Research on population aging serves as a reference for policy decision-making and provides information, data and theoretical frameworks that offer technical support. Studies of population aging can help in the formulation of scientific aging policies and assist in finding solutions to cope with social problems created by an aging population.

\section{The development process and representative achievements of studies on population aging since the reform and opening up}

Since 1978, as China's population has changed and policies have been adjusted in response, research on population aging has expanded steadily and offered richer insights, both theoretical and practical. To explore the developmental progress of studies on population aging during the last 40 years, we used "aging" as the keyword to retrieve Chinese literature for the years 1978-2018 from the bibliographic database CNKI. We retrieved 22,389 references from CNKI, including 16,655 articles published in journals and 5734 masters and doctoral theses. We then analyzed the basic situation of the literature.

The results of literature analysis showed that there are three aspects to the basic characteristics of studies on population aging. Firstly, the number of studies on aging has increased year by year (Fig. 1). Breaking this down, the proportion of studies in journals showed a declining trend, while the proportion of masters and doctoral theses increased. This indicates that individuals pursuing advanced degrees are paying more attention to studies on population aging, and that colleges and universities have gradually become the main force driving research on population aging. Secondly, funding support for studies on population aging increased year by year. The proportion of studies on population aging that received funding gradually increased from $1.3 \%$ in the first stage (1978-1999) to $3.8 \%$ in the second stage (2000-2009) 


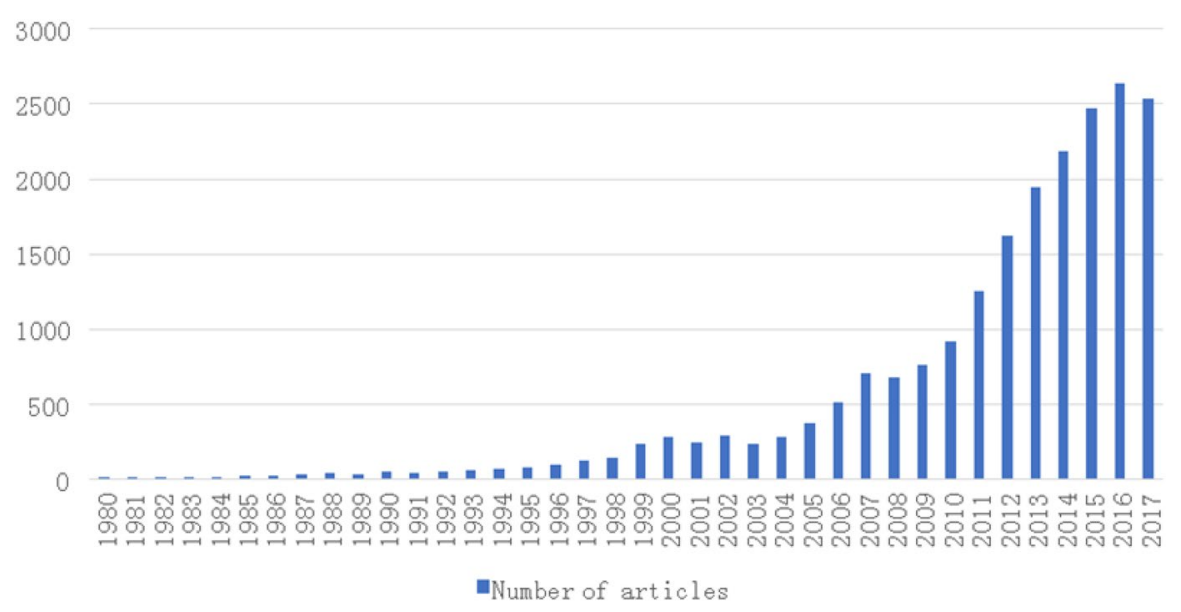

Fig. 1 The quantitative distribution of research on population aging literature in CNKI since 1978

and to $9.6 \%$ in the third stage (2010-2018). This is evidence that the state and the society are paying increased attention to studies on population aging. Finally, the disciplines undertaking studies on population aging have remained constant for the last 40 years. The disciplines engaged in studies on population aging can be divided into four categories, namely sociology, economics, political science and medical science. Among these, proportions of studies from sociology and economics have increased year by year.

During the last 40 years, as the process of aging has changed, the focus of studies on population aging has changed as well; three phases are discernible: studies of aging trends and causes, studies of aging characteristics and policy responses, and studies of aging top-level design and the development of integrated interdisciplinary research efforts. It should be emphasized that the phases describe the general focus of research at different times; they do not represent absolute or arbitrary divisions. While the phases indicate which research areas were prevalent at different times, studies of some topics can be found in each of the phases. Moreover, with the passage of time, new research concepts are constantly being introduced, and research topics on population aging are being continuously updated and enriched.

\section{Phase one: the study on the trends and causes of population aging (1978-1999)}

During the years of 1978-1999, China had yet to become an aging society. After the World Congress on Aging in 1982, scholars began to pay attention to the study of population aging by analyzing the current situation and the development trends of China's population structure. At this stage, the main research topics included aging theory and concepts of aging, analyses of the causes of aging, characteristics, trends, 
strategic countermeasures to cope with population aging, and studies of international experiences for comparison and reference on aging.

Analyzing aging theories and concepts was an important part of research on population aging, helping to define the direction of work on aging and the formulation of national development strategy (Tao 1997). With this in mind, Mu (1998) analyzed the basic concept of ageing and put forward an original meaning of "aging". He argued that aging referred to the aging process or aging phenomenon. The problems of the elderly needed to be understood comprehensively, not only the problems of old people but also problems with the environment in which the elderly lived. In short, addressing the aging problem requires the construction of an old-age support system and an elderly assistance service system that extends from families to national level programs. The concepts of "healthy aging", "successful aging" and "productive aging" were introduced to China after being put forward by the international community; these concepts received extensive attention from academic circles in China (Wu 1997; Tao 1998). In addition, the study of aging trends of the population requires that we first pay attention to the relevant indicators of the aging population and the criteria that indicate entry into an aging society. Based on his study of international experiences, $\mathrm{Wu}$ and $\mathrm{Du}$ (1992) suggested that when the proportion of the population aged 65 and above reaches or exceeds $7 \%$ of the total population, the society is an aging society. If there is no data on the population aged 65 and above, data for the proportion of the population 60 and above should be used, and the standard of $7 \%$ should be adjusted accordingly.

To identify the causes of China's aging problems, scholars started with an examination of China's national conditions and found that the substantial, continuous decline of fertility rate due to promotion of the family planning policy was the root cause of the rapid aging of China's population (Qu 1989; Du 1995). In addition, the significant decline of population mortality and the increase of average life expectancy were also important reasons for the increase of the elderly population (Gu 1997). By analyzing China's population data, Wu and Xu (1990) determined that the characteristics of China's aging population in this stage included: a large population base and rapid economic development, uneven development in different regions, underdeveloped economies, the poor quality of the elderly population, and the effects of population policy.

In addition to studying the causes and characteristics of population aging, scholars also actively learned from international aging experiences. For example, Chen (1991) briefly introduced the aging situation and the re-entry of some elderly people into the labor force in the US, and Yin (1999) made a systematic study of the situation of Japan's aging population and the countermeasures adopted to deal with the problems of aging. It is important to note that there were significant differences between China and other countries during this phase. China had to cope with the challenges created by the aging of the population while continuing to implement the one-child policy for controlling population growth at the same time. Although China should refer to the experiences of other countries, we should proceed from our own national conditions and avoid blindly copying Western experiences. In this regard, $\mathrm{Wu}$ and $\mathrm{Xu}$ (1990) argued that controlling population growth and coping with population aging should go hand in hand, with neither being neglected. The aging 
problem was ultimately treated as a development problem, and improving labor productivity and accelerating economic development was the fundamental guarantee for coping with the problems created by an aging population.

\section{Phase two: the study of population aging characteristics and policy responses (2000-2009)}

In 2000, China formally became an aging society. The country and the society began to pay more attention to the aging population. In 2000, the CPC Central Committee and the State Council issued "The Decision on Strengthening the Work on Aging". In 2006, the development of programs to address issues related to population aging were included in China's 11th Five-Year Plan. During this phase, the main research topics focused on the characteristics and trends of population aging, countermeasures to address issues related to population aging in the twenty-first century, and the influence of aging on the old-age security system (including insurance and medical treatment).

Zeng (2001) pointed out that the main characteristics of China's aging population in the first half of the twenty-first century are "two high and three large". "Two high" refers to high speed growth of the elderly population and high or advanced age of the elderly people, and "three large" refers to the large number of old people, the large dependency ratio of old people and the large regional differences. Wu et al. (2004), Tao (2006) and other scholars also analyzed the main characteristics of China's aging in twenty-first century. These studies found the key characteristics included the large size of the elderly population, the rapid aging process, unbalanced regional development, the fact that rural areas are aging significantly faster than urban areas, and that people were getting old before getting rich. In addition, research institutions predicted the secular trends of aging in the twenty-first century. The National Working Committee Office on Aging has determined that China's population aging trend can be split into three stages during the years from 2001 to 2100: the rapid aging stage (2001-2020), the accelerated aging stage (2021-2050) and the severe aging stage (2051-2100). According to the Office's prediction, China's aging population aged 60 and above will reach a peak of 437 million people in 2051 (The National Working Commission on Aging 2007). The China Research Center on Aging has also divided the aging process into three stages: the transition stage (1982-2000), the development stage (2000-2025) and the peak stage (2025-2055). They argue that the population aged above 60 will increase to 440 million in the peak period (Tao 2006). Based on data from China's 2000 census, the Institute of Gerontology of Renmin University of China predicts that China's population will continue aging during the next 100 years, with the population aged 60 and above peaking at 430 million in the year 2055 (Du et al. 2005). From the above studies, we can see that China's aging population will continue to grow at a rapid rate in the twenty-first century, reaching a peak size around 2050 and then decreasing.

After China formally became an aging society during this stage, questions concerning how to use public policies to deal with population aging problems became hot research topics. Research focused on the old-age security system that included an 
old-age insurance system and a medical insurance system. The rapid aging of China's population put great pressure on the traditional old-age security system that relied, to a great extent, on families to provide care for elderly family members, and the dependency ratio of the elderly population increased as a result. The old-age insurance fund faced a crisis, while at the same time, services providing old-age security and care were seriously lacking (Ling 2009). The shortcomings of the old-age insurance system and the medical insurance system exposed these problems. With respect to the retirement insurance system, as the aging process intensified, the ratio of the retired workers receiving pension payments to in-service workers contributing to the pension fund increased steadily. This affected the balance of the pension fund in China (Zuo 2001). After examining reforms of old-age insurance systems in other countries with aging populations, scholars suggested reform countermeasures for the old-age insurance system in line with China's national conditions. Wang and Lin (2005), for example, investigated pension system reform experiences in the US, Japan, Britain, Israel, Chile and other countries with aging populations, summarized successful international pension reform models, and put forward several policy suggestions for pension system reform tailored to the needs of the pension system in China.

Based on the characteristics and development trends of China's aging population, Zhang (2005) proposed important measures to cope with the problems associated with an aging population. He supported the development of a multi-level retirement insurance system, reforms and improvements to the current system for urban workers, and suggested plans to solve the transition cost of reforming the retirement insurance system, raise the statutory retirement age and create employment opportunities for the elderly. In view of the existence of dual systems for urban and rural areas in China, Liu (2008) argued that the establishment of an integrated urban-rural old-age insurance system was needed to address the fact that some social groups were not covered by any old-age insurance system. He urged implementing integrated management of basic urban and rural old-age insurance at the earliest possible date. With respect to the medical insurance system, the aging of people covered by insurance has increased expenditures and reduced the reserves in the medical insurance fund. These developments are putting the sustainable, stable operation of medical insurance system at risk. He et al. (2009) discussed the "system aging" of basic medical insurance programs, and proposed countermeasures and ideas for health insurance strategies. These strategies included the increase of the statutory retirement age, the establishment of financing mechanisms for retiree medical treatment insurance premiums, expanding basic medical insurance coverage, clearly defining which entities are responsible for funding medical insurance programs, and establishing a basic medical insurance fund reserve.

\section{Phase three: the study of top-level design and interdisciplinary integration on population aging (since 2010)}

As the process of population aging has accelerated, the growing size of the aging population impacted various aspects of life in China, such as politics, economics, social life and culture. Increasingly, people acknowledged the complexity of the 
aging problem and given this complexity, recognized the need for multidisciplinary attention and research of the aging problem. During this stage, the main research topics included top-level design research and interdisciplinary studies focused on aging.

The particular characteristics and severity of China's aging problems made it imperative to strengthen the central government's top-level design of national strategy and policies in order to respond effectively to the challenges of population aging. The basic structure of top-level design for aging problems should be "basic state policy + medium and long-term plans + basic regulations + programme of aging". Firstly, efforts to deal actively with population aging should be raised to the level of basic state policy. Secondly, medium and long-term plans to cope with the problems of aging should be developed as quickly as possible, while at the same time, efforts to strengthen the top-level design of basic livelihood programs such as pensions and the medical security system for the elderly should continue. Finally, reform of the aging work system ( $\mathrm{Li}$ 2013) should be accelerated. Since 18th CPC National Congress in 2012, the party and the government have begun to attach great importance to aging programs. Multi-dimensional reforms of industries and business sectors serving the aging population have been launched, based on a series of principles and policies developed to cope with aging issues. These reform measures have resulted in a series of achievements (Lu 2018a, b). Top-level design must be based on solid research on aging. More studies on aging that explore ideas about how best to undertake the top-level design of aging efforts are needed.

Inevitably, research on population aging has moved in the direction of interdisciplinary studies. Research into the aging phenomenon and process must be both comprehensive and deep. As early as 1969, the International Association of Gerontology proposed that the four pillars of gerontology were biology, clinical medicine, psychology and sociology (Du and Wu 2001). Nowadays, studies on aging can be divided into social science studies and natural science studies. Social sciences disciplines engaged in studies on aging include economics, political science, ethics, psychology, law, education, etc. Research contents include social economic conditions of the elderly, issues concerning equal treatment for the elderly, legal protections for the elderly, crime problems, and mental health of the elderly. For example, Feng and Chen (2017) presented a detailed analysis of the intermediary effects aging has on economic growth. He found that population aging can affect the economic growth rate through its impact on the labor productivity rate, labor participation rate and proportion of working-age population. Studies on aging produced by natural science disciplines include research on clinical medicine, nursing, medical practices, biology as it relates to the elderly, architecture, etc. Research contents include the diagnosis and treatment of the elderly diseases, elderly nursing practices, the relationship between genetics and aging, elderly biomedical indexes, and studies of architecture for the elderly. For example, Geng and Zhao (2018) explored the relationship between ACE gene I/D polymorphism and successful aging of people over 80 years old, and analyzed the interaction between gene and lifestyle to provide a basis for an intervention study of successful aging.

Since this phase began in 2010, demographic experts have gradually begun to appreciate the importance of interdisciplinary research on the health of the elderly. 
Scholars have started paying more attention to whether the increased size of the elderly population and rising life expectancy are accompanied by an increase or decrease in the health level of the elderly. At present, there are three theoretical hypotheses held by different scholars: the theory of disease and disability compression, the theory of disease and disability expansion, and the theory of dynamic equilibrium. The theory of disease and disability compression argues that with the development of the social economy and improvements to medical care services, health levels and living standards, elderly people can delay the onset of disabilities and chronic diseases while extending their life spans (Fries 1980). The theory of disease and disability expansion holds that the extension of elderly life spans includes increased life expectancies for both healthy and infirm elderly, resulting in an increase in the proportion of the elderly with disabilities (Olshansky et al. 1991). Finally, some scholars believe that these two phenomena might cancel each other out, resulting in a situation characterized by a dynamic balance (Manton 1982). At present, there is no agreement on which theoretical hypothesis is better. Research and intervention of the society into the factors affecting the health of the elderly is needed. As a result, China has begun to pay attention to the development of interdisciplinary research focused on the health of the elderly.

Since the individual life spans and health status of human beings are determined by the interaction of social, behavioral, environmental and genetic factors, if we only explore the influence of genes on the health of old people from the perspective of medical biology, we cannot clearly understand the effect of genes on health under specific environmental and behavioral conditions. Therefore, the development of interdisciplinary research on the health of the elderly should be carried out, and scholars from the fields of medicine, genetic biology and the social sciences should come together to study the influence of social, behavioral, environmental and genetic factors and their interactions on the health of the elderly (Zeng 2011; Lu 2018a, b). Zeng et al. (2014), for example, reviewed the progress of international research on the impact of environmental and genetic interactions on elderly health, summarizing three relevant earlier case studies that concerned three genetic types, FOXO, ADRB2-rs1042718/ADRB2-rs1042718 and APOE4. These studies revealed the influences of genes on elderly health and concluded that if health interventions gave full consideration to the interactions of environmental factors and genetic types, we could greatly improve the efficiency of the elderly health interventions. Although there have been many research papers about longevity genes, the majority of these paper discussed the problems only from the single perspective of genetics. Medical biologists were unable to combine their research on genetic factors with data on social behavior and environmental factors, and sociologists lacked biological research experience. Such limitations were not conducive to the development of interdisciplinary health research on aging (Zeng 2012). In the future, great attention should be paid to the research across natural and social sciences on the health of the elderly. By incorporating genetic testing data into aging follow-up surveys, the influence of social, behavioral, environmental, and genetic factors, and their interactions on elderly health can be explored. 


\section{The main characteristics and future prospects of studies on population aging}

A review of the development of research on population aging during the last 4 decades of reform and opening up in China shows that studies on aging started with explorations of aging practices, theories and definitions and finally moved to studies of the main characteristics of aging and the mechanisms influencing the aging society in the twenty-first century. Recently, as the pace of aging in China has accelerated, studies on population aging have focused on the old-age pension system and often feature interdisciplinary cooperation. China's studies on population aging have made worthwhile achievements.

\section{The main characteristics of studies on population aging}

Since China's reform and opening up began, the following five aspects have characterized China's studies on population aging.

First is the combination of macro and micro perspectives. The research on population aging not only includes studies of changes to China's population structure, reports on centennial development trends of aging and papers on the reform of the old-age security system from a macro perspective, but also includes research on regional old-age care issues from the micro perspective. The range of research contents and perspectives is wide indeed.

Second is the combination of theory and practice. The research on population aging applies international aging concepts to China's practices, which promote an understanding of the aging society that takes into consideration China's national conditions. Moreover, research on population aging in China also closely tracks national policy trends and the social demand.

Third is the fact that much research on population aging concerns topics that are also hot social issues. Some of these issues include studies of "empty-nest" households (i.e., elderly individuals or couples living alone without their children nearby), the pension insurance system, and the integration of medical care and nursing care. The hope is that the research results are practical and forward-looking, and can applied in a timely manner to address the most urgent social problems.

Fourth is the combination of quantitative and qualitative research. Studies on population aging not only adopt quantitative methods to analyze the causes, characteristics, trends and influences of aging in China, but also use qualitative methods to study the social problems arising from the aging process, such as issues concerning rural old-age support and delayed retirement.

Fifth is the combination of international vision with localization suitable for China. China was relatively late to begin studying population aging. Thus, our first task was to learn about the social situation and research progress in other countries, and then later combine these international insights with China's national conditions to address the problems of China's aging society. 


\section{The future prospects of the studies on population aging}

The future will be a period of rapid changes in China's population structure, as well as a period during which the problems of aging become more acute. Therefore, studies on population aging must be rooted in China's basic national conditions, taking into consideration social and economic development to highlight the diversity, fundamentality and foresight of the studies on population aging. There are five specific ways in which China's studies on population aging should develop in the future.

First, research must actively build a theoretical system of aging with Chinese characteristics. In the future, based on actively learning from theoretical achievements on aging internationally, we should strengthen the theoretical framework supporting China's practical research and actively construct a theoretical system of aging with Chinese characteristics. Second, there must be more basic research to lay a solid foundation for theoretical development. China's aging problem is relatively complex, and in the future, we should produce more in-depth research on the multiple social impacts that accompany aging. Third, we must strengthen the development of interdisciplinary research on population aging and break away from narrowly focused disciplinary studies. Due to institutional arrangements, natural and social sciences research takes place on separate paths. As a result, China's studies on population aging are hindered by a lack of interdisciplinary cooperation, and this is not suitable for the key needs of the country and society. Therefore, it is necessary to remove the limitations, promote close cooperation between disciplines and strengthen the development of interdisciplinary research on population aging in the future. Fourth, policies should be closely linked with the people's needs, and close attention should be paid to the practicality and foresight of research on population aging. In addition to establishing a theoretical system of aging with Chinese characteristics, research results on population aging which are rooted in theory should be developed and applied in practice. Fifth, the timely sharing of survey data and research results among various research institutes should be strengthened. In the future, a normative research on population aging database should be established to promote effective communication of data and resources. At the same time, we should use scientific and technological means to build a cloud platform for big data on aging, and fully make use of science and technology in studies on population aging, so as to promote the sustainability of studies on population aging in China.

\section{References}

Chen, Q. (1991). America's population aging and elderly population reemployed status. Population Journal,4, 58-61. (in Chinese).

Du, P. (1995). China's fertility rate declining and aging population. Chinese Journal of Population Science, 2, 12-17. (in Chinese).

Du, P., \& Wu, C. (2001). Interdisciplinary research and the development of gerontology in the 21st century. Journal of Renmin University of China,3, 68-69. (in Chinese).

Du, P., Zhai, Z., \& Chen, W. (2005). Centennial development trend of China's population aging. Population Research, 6, 90-93. (in Chinese). 
Feng, J., \& Chen, W. (2017). Analysis on the mechanism of population aging affecting economic growth in China: From the perspective of mediating effect. Population Journal,39(4), 93-101. (in Chinese).

Fries, J. (1980). Aging, natural death, and the compression of morbidity. The New England Journal of Medicine,303, 130-135.

Geng, X., \& Zhao, L. (2018). Relationship between ACE gene I/D polymorphism and successful aging of population over 80 years old. In Proceedings of the 2018 session of the sports physiology committee of the Chinese physiological society and the symposium on "scientific and technological innovation and exercise physiology", pp. 65-67 (in Chinese).

Gu, Y. (1997). The particularity of China's aging population and the diversification of pension modes. Population Journal,1, 21-25. (in Chinese).

He, W., Xu, L., Fu, K., Liu, X., \& Yang, Y. (2009). Study on "system aging" of basic medical insurance and countermeasures. Chinese Journal of Population Science, 2, 74-83. (in Chinese).

$\mathrm{Li}, \mathrm{Z}$. (2013). The top-level design of population aging from four areas to start. China Economic Herald,2013, B07. (in Chinese).

Ling, W. (2009). The challenges and countermeasures of the aging population to the pension system. Seeker,10, 81-83. (in Chinese).

Liu, T. (2008). The exploration of establishing an urban and rural integral old-age insurance system under the background of population aging. Shandong Social Sciences, 1, 35-38. (in Chinese).

Lu, J. (2018a). Prospect of interdisciplinary health research on aging in the new era. China Social Work Research,8(middle), 30-31. (in Chinese).

Lu, J. (2018b). The main idea and strategic conception of the top-level design for coping with population aging in the new era. Population Research,1, 21-26. (in Chinese).

Manton, K. (1982). Changing concepts of morbidity and mortality in the elderly population. The Milbank Memorial Fund Quarterly Health and Society, 60, 183-244.

$\mathrm{Mu}, \mathrm{G}$. (1998). The aging of population and the issue of aging-answer Comrade Tao Liqun. Population Journal,1(3-7), 57. (in Chinese).

Olshansky, S., Rudberg, M., Carnes, B., et al. (1991). Trading off longer life for worsening health: The expansion of morbidity hypothesis. Journal of Aging and Health,3, 194-216.

Qu, H. (1989). Demographic reasons for China's aging population. Population Research,4, 8-16. (in Chinese).

Tao, L. (1997). On the analysis of population aging-discussing some theories and concepts about population aging with Comrade Mu Guangzong (one). Population Journal,6, 9-20. (in Chinese).

Tao, L. (1998). On the analysis of population aging-discussing some theories and concepts about population aging with Comrade Mu Guangzong (two). Population Journal,1, 8-11. (in Chinese).

Tao, L. (2006). The trend and characteristics of China's population aging. Scientific Decision Making,4, 8-10. (in Chinese).

The National Working Commission on Aging. (2007). China's Aging Population Trend Forecast Research Report. Chinese Women's Movement,2, 15-18. (in Chinese).

Wang, G., \& Lin, Z. (2005). The reform mode of foreign pension system under the challenge of "population aging" and its reference. Population Journal,2, 21-25. (in Chinese).

Wu, C. (1997). Building a healthy aging society - the right choice for China to embrace the aging of the 21st century. Population Journal,1, 2-6. (in Chinese).

Wu, C., \& Du, P. (1992). Recognition of the aging trend of China's population. Chinese Journal of Population Science, 3, 1-5. (in Chinese).

Wu, C., \& Xu, Q. (1990). A new understanding of the trend and characteristics of China's population aging and new thinking on strategic countermeasures. Chinese Journal of Population Science,2, 12-17. (in Chinese).

Wu, C., Wang, L., \& Miao, R. (2004). The process, prospect and countermeasures of population aging with Chinese characteristics. Population Research, 1, 8-15. (in Chinese).

Yin, H. (1999). Japan's population aging and countermeasures. Population Journal,6, 17-21. (in Chinese).

Zeng, Y. (2001). The characteristics and countermeasures of China's aging population. Population and Economics, 5, 3-9. (in Chinese).

Zeng, Y. (2011). The international development of interdisciplinary study of factors affecting the health of older people. Chinese Science Bulletin,56(35), 2929-2940. (in Chinese).

Zeng, Y. (2012). Interdisciplinary study of health in old age: Social, behavioral, environmental, and genetic factors and their interactions. Chinese Journal of Health Policy, 5, 5-11. (in Chinese). 
Zeng, Y., Chen, L., Ruan, R., Chen, H., Li, J., Zhang, F., et al. (2014). The influence of the interaction between environment and genetic factors on the health of the aged-a review of previous studies. Medicine and Philosophy (A),35(9), 1-25. (in Chinese).

Zhai, Z., Chen, J., \& Li, L. (2017). China's population and aging trend from 2015 to 2100. Population Research,41(4), 60-71. (in Chinese).

Zhang, Y. (2005). Population aging and China's pension insurance system reform. Journal of Sichuan Normal University (Social Sciences Edition),2, 18-23. (in Chinese).

Zuo, X. (2001). China's pension security facing the population aging: Challenges and policy choices. Chinese Journal of Population Science,3, 1-8. (in Chinese).
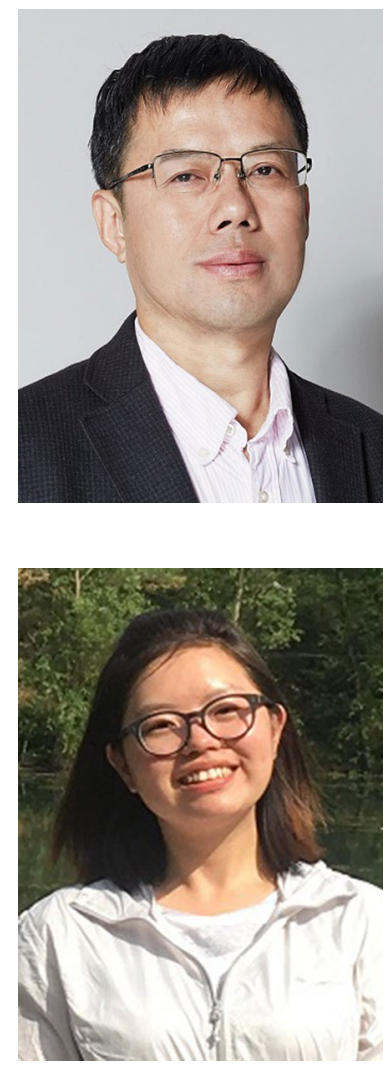

Jiehua Lu is a Professor at Department of Sociology, Peking University. His academic field is demography and gerontology.

Liu Qin is a Ph. D student at Department of Sociology, Peking University. Her research interests are population health and population aging. 